\title{
DC Characterization of the Coaxial Superconducting Cable
}

\author{
J. Šouc, F. Gömöry, M. Vojenčiak, L. Frolek \\ Institute of Electrical Engineering, Centre of Excellence CENG, SAS \\ 84104 Bratislava, Slovakia \\ D. Isfort, J. Ehrenberg AND J. Bock \\ Nexans SuperConductors GmbH \\ Chemiepark Knapsack 50351, Hürth, Germany \\ Coaxial cable model with superconducting core and superconducting \\ shield conductor was constructed and tested in DC regime. While the core \\ was already examined in our previous works, in this contribution the de- \\ tailed study of the superconducting shield conductor in DC conditions is \\ presented. It consists of $16 \mathrm{ReBCO}$ coated tapes with critical current $35 \mathrm{~A}$ \\ each connected in parallel. Using shunts with known values placed in series \\ the currents in individual tapes were possible to measure. Distribution of the \\ total cable current into the individual tapes was monitored and its influence \\ on critical current of the cable is discussed.
}

PACS numbers: 74.25.Sv, 74.25.Fy, 84.71.Mn

\section{Introduction}

Transmission power superconducting cable lines are considered to use in one of the following configuration: as three independent phases with three cryogenic cryostats, as three phases in one cryogenic envelope or as three concentric phases (triaxial) placed in one cryostat. Every solution has its advantage. In the case of coaxial phases no outer magnetic field exists, low ac loss is generated and very low cable inductance is achieved.

In this work we present experiments with short model of such kind of cable, in particular the test in $\mathrm{DC}$ regime.

\section{Experimental}

Superconducting coaxial cable model consisted of two parts: superconducting core and superconducting shield conductor. The core was constructed of 14 YBCO tapes of 75 A critical current [1]. Tapes were placed in single layer on the surface of the epoxy-fiberglass mandrel of diameter $2 \mathrm{~cm}$ and length of $50 \mathrm{~cm}$, with the lay angle of $15^{\circ}$. The electrical connection between each tape and the cable ter- 
mination was created by soldering of 4 flexible braids consisting of $\varnothing 80 \mu \mathrm{m} \mathrm{Cu} / \mathrm{Ag}$ wires as is shown in lower part of Fig. 1a. Such unusual technology was utilized because only $2 \mu \mathrm{m}$ metallic protection layer of the YBCO tapes was used. Total transport capacity of the superconducting tapes in the core was $900 \mathrm{~A}$. Complete DC characterization of this cable part has been already performed in our previous work [2]. The core was inserted into the shield conductor and both conductors were connected in series by $\mathrm{Cu}$ plate on one cable side; to the other cable side $\mathrm{Cu}$ strands for current supplying were attached. The shield conductor was constructed from 16 ReBCO coated tapes of $10 \mathrm{~mm}$ width [3]. They were placed in parallel configuration on the surface of the epoxy-fiberglass mandrel of diameter $10 \mathrm{~cm}$ and length of $50 \mathrm{~cm}$ in one layer, with the lay angle of $15^{\circ}$ - upper part of Fig. 1a. Connection to the $\mathrm{Cu}$ terminals was performed by soldering.
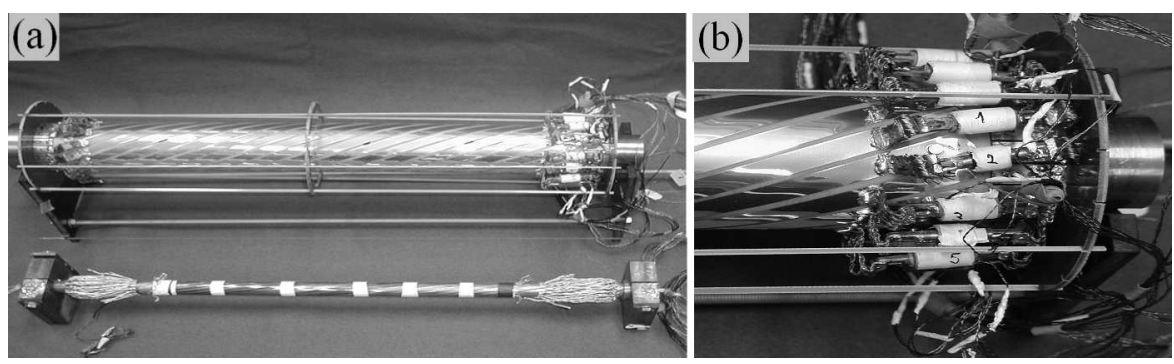

Fig. 1. (a) Lower part - superconducting core, upper part - shield superconducting conductor. (b) Detail of the shield superconducting conductor with calibrated resistors for measurement of the currents in individual tapes.

To each tape the calibrated resistor was connected in series allowing to measure the current $I_{m}$ of individual tapes — Fig. 1b $(m=1-16)$. In such way the distribution of the total cable current $I_{\text {tot }}$ was possible to monitor. On each tape the voltage taps were placed to measure the voltage on individual tapes $U_{m}$. The distance between taps was $l=60 \mathrm{~cm}$. From measured $I_{n}$ and $U_{m}$ the $I-V$ characteristics of all tapes forming the shield conductor were created. More, the total voltage $U_{\text {tot }}$ of the shield conductor was measured on the current terminals allowing to derive its $I-V$ characteristic. KEYTHLEI 2700 voltmeter was used for measurement of all 34 measuring points in one run.

\section{Results and discussion}

Critical currents $I_{\mathrm{cm}}$ of the individual tapes were determined from their $I-V$ characteristics shown in Fig. 2a. They were approximated by power-law model $E_{m}=E_{\mathrm{c}}\left(I_{m} / I_{\mathrm{c} m}\right)^{n}$, where $E_{m}=U_{m} / l, E_{\mathrm{c}}=1 \mu \mathrm{V} / \mathrm{cm}$ represents the criterion for critical current $I_{\mathrm{cm}}$ estimation and $n$-value denotes the slope of the $U_{m}$ increasing in dependence on $I_{m}$. The scatter of $I_{\mathrm{c} m}$ estimated in this way is rather high - from $28 \mathrm{~A}$ up to $45 \mathrm{~A}$. On the other hand, the tapes can carry the 

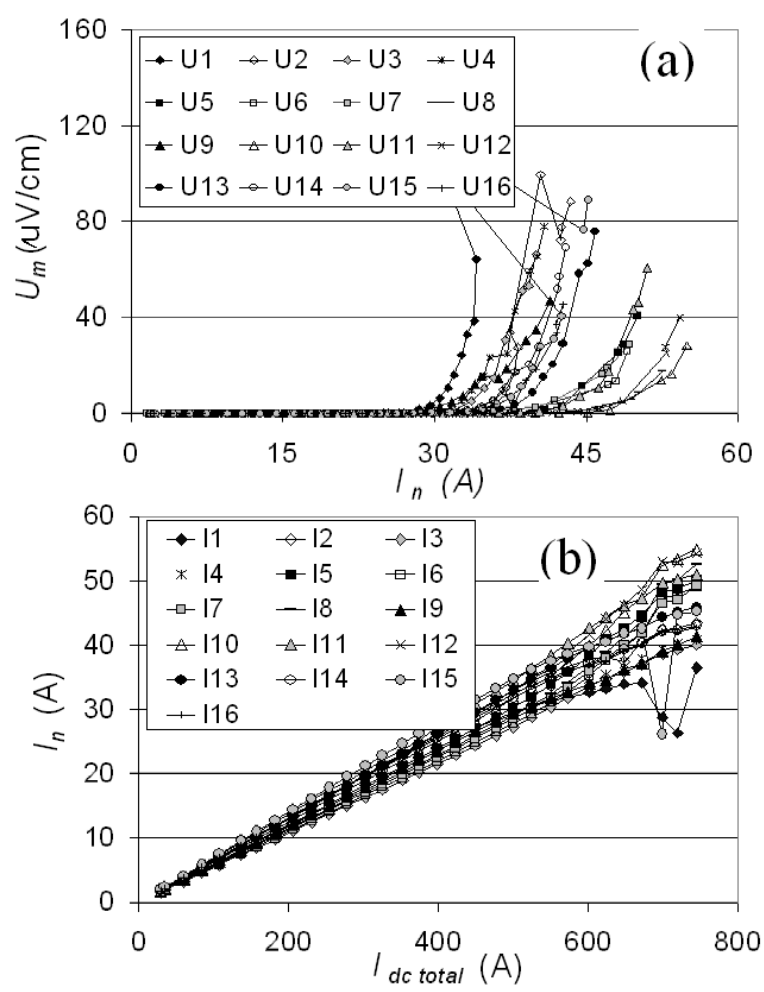

Fig. 2. (a) $I-V$ characteristics of the individual tapes, (b) currents in individual tapes in dependence on total cable current.

currents raising $E_{m}$ respond by several tens of microvolt. This property of the tapes is required for real cable stability in the case of over-critical current regime.

In Fig. 2b the dependence of the currents $I_{m}$ flowing in individual tapes on total cable current $I_{\text {tot }}$ is shown. Current distribution to the individual tapes is determined by linear resistances (electrical contacts of the tapes to the current terminals and calibrated resistor in individual paths) and by non-linear properties of the superconducting tapes itself [4]. In discussed cable model the small non-homogeneity in $I_{m}$ distribution was ensured by soldering of the calibrated resistor to the current terminal in contrast to the cable model described in [4] where the shunts were mechanically connected by screws.

The dependence of total voltage $U_{\text {tot }}$ on total current $I_{\text {tot }}$ of the cable is presented in Fig. 3. This voltage is a sum of the potential drop on superconducting tapes $U_{\mathrm{sc}}$ and voltage on the resistive parts $U_{\mathrm{Cu}}$. Resistive part $R_{\mathrm{Cu}}$ was calculated from linear fraction of the $U_{\text {tot }}\left(I_{\mathrm{tot}}\right)$. Thus for determining of the $I-V$ characteristic of the cable the voltage on the superconducting part was taken as difference $U_{\mathrm{sc}}=U_{\mathrm{tot}}-I_{\mathrm{tot}} R_{\mathrm{Cu}}$. As was shown in [5] the correct $I-V$ characteristic of the cable can be derived also by averaging of the voltages of the individual tapes at 


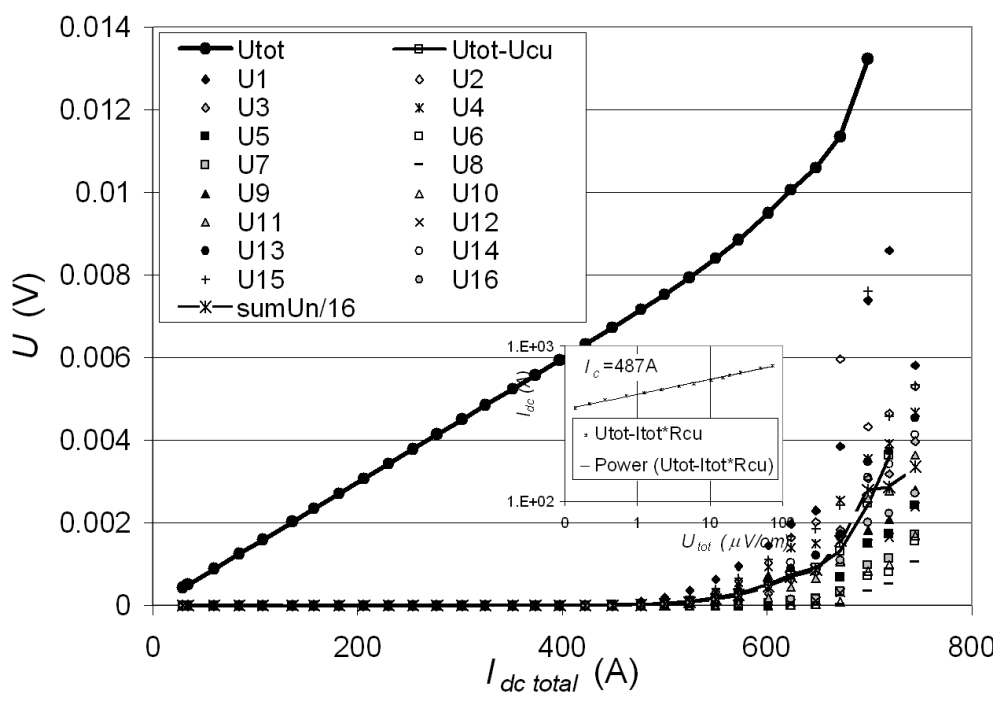

Fig. 3. Dependence of the total cable voltage $U_{\text {tot }}$ measured on current terminals on total cable current $I_{\text {tot }}$. The inset shows $I-V$ characteristic of the cable in log-log representation - power dependence typical of superconductors was found.

certain total current. Both of them are depicted in Fig. 3 and good coincidence was found.

\section{Conclusion}

Short coaxial cable model with superconducting core and shield conductor was designed and constructed from ReBCO coated superconducting tapes. $I-V$ characteristics of the individual tapes as well as the outer shield conductor were measured. Distribution of the total current to the individual tapes was checked as well.

\section{Acknowledgments}

This work was supported in part by the European Commission (project SES6-CT-2004-502615 "Super3C") and Grant Agency APVV (contract APVV51-45605).

\section{References}

[1] A. Usoskin, A. Rutt, J. Knoke, H. Krauth, T. Arndt, IEEE Trans. Appl. Supercond. 15, 2604 (2005).

[2] J. Šouc, F. Gömöry, B. Klinčok, L. Frolek, M. Vojenčiak, A. Usoskin, A. Rutt, in: Applied Superconductivity Conference, Seattle (USA) 2006, No. 4LW04-CE3846.

[3] Nexans SuperConductors GmbH, Chemiepark Knapsack 50351, Hürth, Germany.

[4] L. Frolek, F. Gömöry, Physica C 401, 227 (2004).

[5] L. Frolek, F. Gömöry, J. Šouc, Czech. J. Phys. 54, Suppl. D, D497 (2004). 